\title{
Technology-Based Psychosocial Interventions for People with Borderline Personality Disorder: A Scoping Review of the Literature
}

\author{
Álvaro Frías ${ }^{a, b}$ Laia Solves $^{a, b}$ Sara Navarro ${ }^{a, b} \quad$ Carol Palma $^{a, b}$ \\ Núria Farriols $^{a, b}$ Ferrán Aliaga $^{a, b}$ Mònica Hernández ${ }^{b}$ Meritxell Antón ${ }^{b}$ \\ Aloma Rierab \\ ${ }^{a}$ Facultat de Psicologia, Ciències de l'Educació i de l'Esport Blanquerna, University of Ramon-Llull, Barcelona, Spain; \\ ${ }^{\mathrm{b}}$ Adult Outpatient Mental Health Center, Consorci Sanitari del Maresme, Hospital of Mataró, Mataró, Spain
}

\section{Keywords}

Borderline personality disorder · Software · Clinical research . Technology-based interventions · Psychosocial treatments

\begin{abstract}
Evidence-based psychosocial interventions for borderline personality disorder (BPD) still face multiple challenges regarding treatment accessibility, adherence, duration, and economic costs. Over the last decade, technology has addressed these concerns from different disciplines. The current scoping review aimed to delineate novel and ongoing clinical research on technology-based psychosocial interventions for patients with BPD. Online databases (PubMed, Cochrane Library, EMBASE, Web of Science, Psyclnfo, and Google Scholar) were searched up to June 2020. Technology-based psychosocial treatments included innovative communication (eHealth) and computational (e.g., artificial intelligence), computing (e.g., computer-based), or medical (e.g., functional magnetic resonance imaging [fMRI]) software. Clinical research encompassed any testing stage (e.g., feasibility, efficacy). Fifteen studies met the inclusion criteria. The main findings were the following: almost two-thirds of the studies (9/15) tested software explicitly conceived as adjunctive interventions to conventional therapy; nearly half of the
\end{abstract}

studies (7/15) were referred to as dialectical behavior therapy-based software; most studies (13/15) were focused on the initial stage of the clinical research cycle (feasibility/acceptance/usability testing), reporting good results at this point; more than one-third of the studies (6/15) tested mobile apps; there is emerging evidence for Internet-based interventions and real-time fMRI biofeedback but only little evidence for mHealth interventions, virtual and augmented reality, and computer-based interventions; there was no computational technology-based clinical research; and there was no satisfaction/preference, security/safety, or efficiency testing for any software. Taken together, the results suggest that there is a growing but still incipient amount of technology-based psychosocial interventions for BPD supported by some kind of clinical evidence. The limitations and directions for future research are discussed.

(c) 2020 S. Karger AG, Base

\section{Introduction}

\section{Rationale}

Borderline personality disorder (BPD) is characterized by pervasive affective, interpersonal, identity, cognitive, and behavioral instability as well as high rates of sui- 
cidal and self-harm behaviors [1]. Its prevalence in the general population is estimated to range from 1.2 to $2 \%$ [2]. Those affected are often severely impaired in their social and professional functioning [3]. BPD also constitutes a high economic burden on society [4].

Several psychosocial treatments for BPD have been developed and positively tested, including dialectical behavior therapy (DBT), schema therapy, mentalization-based therapy, and transference-focused psychotherapy $[5,6]$. These treatments notably take a long time to administer, requiring several years for completion. Some of these patients discontinue therapy in the beginning or abruptly [7]. Meanwhile, implementation and dissemination are slow, and most patients with BPD do not receive these treatments. Within this context, technology-based psychosocial interventions may be an adequate way to overcome treatment barriers and limitations for people with BPD [8].

In general, current developments of healthcare technology for mental health comprise a wide range of disciplines, including, but not limited to: (1) information and communication technology or eHealth: Internet-based treatments (website-based, virtual therapeutic communities); mHealth (mobile app-based, wearable biosensor devices); telehealth (videoconferencing, email messages); (2) computing technology: computer-based; serious games; virtual reality; eye-tracking; (3) computational technology: artificial intelligence; machine learning; deep learning; and (4) medical technology: functional magnetic resonance imaging (fMRI) [9-12]. The integration of these types of technology into the current psychosocial treatments for mental disorders has increased rapidly over the last 10 years, with ongoing clinical research mostly from initial stages of testing (feasibility/acceptance/usability studies) rather than from later stages (efficacy/effectiveness studies). Except for the sound evidence on Internet-based interventions for depression and anxiety disorders [13], such clinical research is providing promising but still not well-established clinical evidence for transdiagnostic psychiatric symptoms (e.g., suicidal ideation) and common mental disorders (e.g., schizophrenia, bipolar disorder, eating disorders, substance use disorders, anxiety disorders, major depression, posttraumatic stress disorder, autism spectrum disorders, attention deficit hyperactivity disorder) [14-16].

As mentioned above, technology-based psychosocial interventions may also be an optimized way to solve potential problems associated with conventional face-toface treatments in people with BPD [8]. The integration of technology into the evidence-based treatments for these patients, either as an adjunctive or as an alternative innovative therapy, can make therapy more accessible, complete, flexible, comfortable, accurate, and ultimately cost-effective. Similar to the aforementioned mental disorders, current clinical research on technology-based psychosocial treatments for BPD is also providing growing but initial clinical evidence [8]. At present, the number of ongoing projects warrants a review to establish an overall vision of this issue.

\section{Objectives}

There were no prior scoping reviews aimed at addressing technology-based psychosocial interventions for people with BPD. Hence, the present paper sought to fill this gap in the literature by performing an up-to-date review of those ongoing studies in BPD that tested any type of technology (as described in the above section) through any stage of the clinical research cycle (from feasibility/ usability/acceptance to efficiency testing).

\section{Methods}

\section{Information Sources}

A literature search was carried out through the PubMed, Cochrane Library, EMBASE, Web of Science, PsycInfo, and Google Scholar databases from inception to June 2020. The terms employed included indexing terms (e.g., MeSH) and free texts: (borderline personality) AND (ehealth OR mhealth OR mobile app OR computer-based OR Internet-based OR technology OR artificial intelligence OR biofeedback OR machine learning OR virtual reality OR telehealth OR serious games OR wearable device OR eye-tracking OR digital OR big data OR fMRI).

\section{Eligibility Criteria}

Studies were selected if they included the following three characteristics: (1) participants: subjects with a main diagnosis of BPD confirmed by semi-structured clinical interviews; (2) interventions: technology-based psychosocial treatments consisting of innovative communication (eHealth) and computational (e.g., artificial intelligence), computing (e.g., computer-based), or medical (e.g., fMRI) software; and (3) outcomes: findings from any stage of the clinical research cycle (e.g., feasibility, efficacy, safety, satisfaction, efficiency). Also, studies were to appear in a peer-reviewed journal and to be accessible in the English language.

\section{Study Selection}

Detailed data of the study selection process are shown in Figure 1. Thirty-six papers were initially collected, but at the end of the process 21 papers had been removed. Thus, 15 papers were included in the current scoping review.

\section{Data Items}

Data from each of the selected papers was separately recruited, classified, and described according to: 


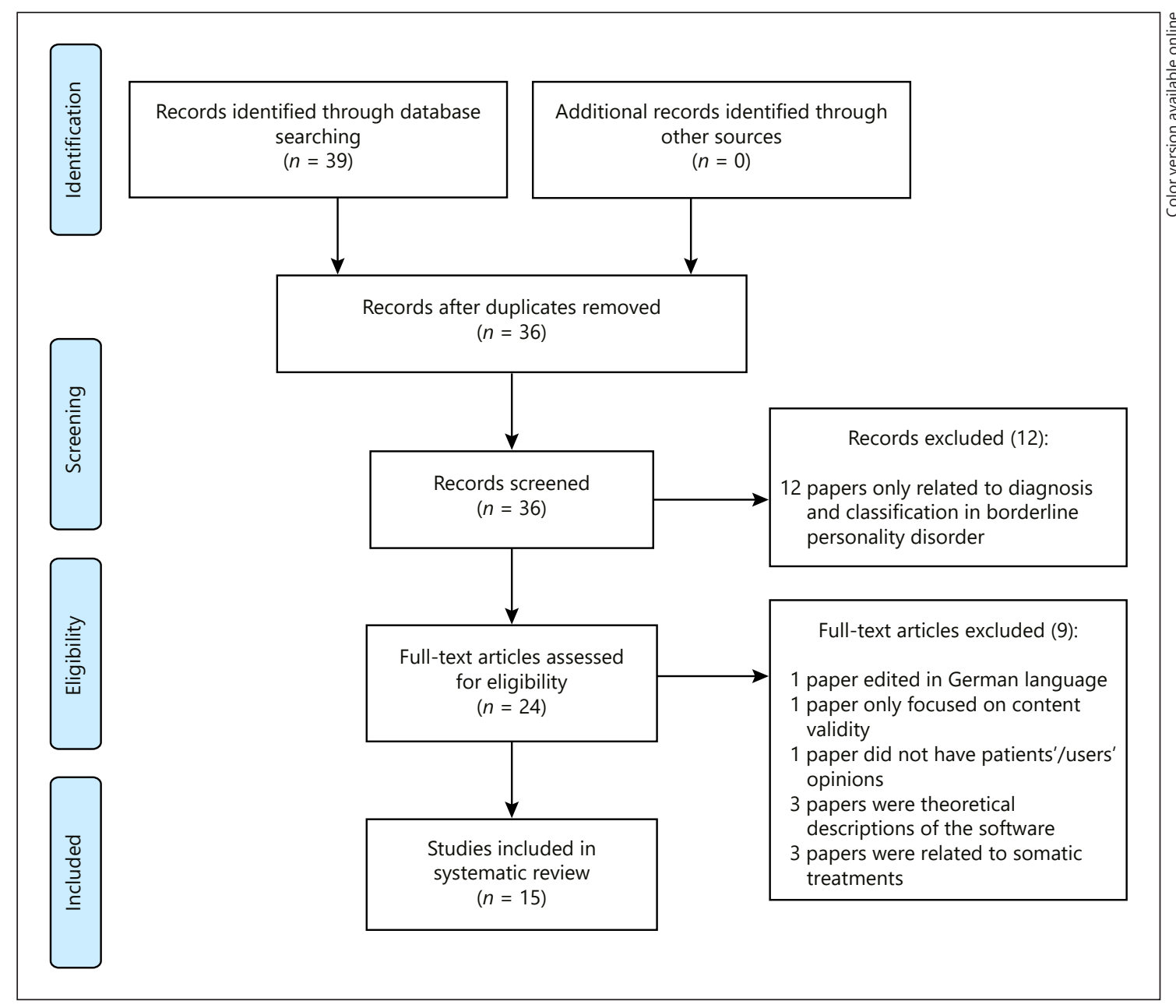

Fig. 1. Flowchart of study selection.

(1) Type of technology-based psychosocial intervention: (a) Information and communication technology: Internet-based (website-based, virtual therapeutic communities); mHealth (mobile app-based, wearable biosensor devices); telehealth (videoconferencing, email messages). (b) Computer technology: virtual/augmented reality; serious games; eye-tracking; computer-based. (Internet-based and computer-based were separately classified because the former does not necessarily need to be installed on the computer. In addition, computer-based technologies were nor run via the Internet.) (c) Computational technology: artificial intelligence; machine learning; deep learning. (d) Medical technology: fMRI.

(2) Type of clinical research: (a) Feasibility/acceptance/usability testing. (b) Efficacy/effectiveness testing. (c) Satisfaction/preference testing. (d) Security/safety testing. (e) Efficiency testing (cost-effectiveness).

\section{Additional Analyses}

The included studies were thought to be too different in type of technology-based psychosocial intervention and type of clinical research. For these reasons, a meta-analysis was not considered as the best choice. Also, a systematic review was ruled out because this was a novel field of research. Accordingly, a scoping review was considered as the more appropriate approach.

A mixed approach to synthesize and categorize the findings was created based on the following classification methods: (1) technology readiness level [17]; (2) software testing research [18]; and (3) evidence-based medicine [19]. This mixed approach was considered better than one of them alone because it comprised different aspects to take into account when analyzing each type of clinical research from technology-, software-, and medicine-based standpoints altogether.

The proposed mixed approach was called the research developmental level (RDL). Papers were scored according to the metrics for each stage of the clinical research cycle listed in Table 1. The total score may range between 0 and 20. Depending on the total score, the RDL of each paper was classified as follows: 0-3 (immature), 4-8 (emerging), 9-12 (promising), 13-16 (adequate), and 17-20 (validated). 
Table 1. Metrics for each stage of the clinical research cycle

\begin{tabular}{lll}
\hline Type of clinical research & & Points \\
\hline $\begin{array}{l}\text { Feasibility/usability/acceptance testing } \\
\text { Feasibility testing } \\
\text { Usability and/or acceptance testing } \\
\text { If based on }\end{array}$ & & 1 \\
& single-case study & 1 \\
& pilot study & 0 \\
\hline Efficacy/effectiveness testing \\
Randomized controlled trial & multiple user groups & 0.5 \\
& & 1 \\
\hline Open trial & if multicenter & 4 \\
\hline Satisfaction/preference testing & if active control treatment & 1 \\
\hline Safety/security testing & & 1 \\
\hline Efficiency testing & valid for efficacy/effectiveness study & 3 \\
\hline Additional metric & valid for efficacy/effectiveness study & 2 \\
\hline
\end{tabular}

Data Collection and Classification Process

Studies were selected by the first two authors of the current paper independently. At the end of the selection process, both researchers had gathered the same 15 papers. Regarding the proposed RDL classification, one of the papers was scored differently by the researchers, but this was clarified after reviewing the study together.

\section{Results}

Detailed data of the results can be seen in Table 2. It includes 15 studies with accurate information based on the following variables: (1) type of technology (e.g., mobile apps, web-based); (2) project name and authors; (3) type of treatment and target symptom (e.g., biofeedback, cognitive remediation); (4) distinctive features (e.g., unguided self-management, beta version); (5) feasibility, usability, and acceptance testing (if performed); (6) efficacy and effectiveness testing (if performed); (7) total score (quantitative measure based on the proposed RDL); and (8) RDL (qualitative data based on the proposed RDL).

\section{Psychosocial Interventions Based on Information and} Communication Technology

mHealth-based Interventions. (1) Mobile app-based interventions. There were five types of software (three beta versions and two full versions) with little clinical evidence (RDL: immature): B.RIGHT [20], Sense-IT [21], EMOTEO [22], DBT Coach $[23,24]$, and mDiary app
[25]. All of them should be used as unguided self-management interventions. Three were DBT-based, to be delivered as an adjunctive tool to conventional DBT therapy (EMOTEO, DBT Coach, mDiary app); another was based on biofeedback using a smartwatch (wearable biosensor device) and was aimed at ameliorating emotional dysregulation (Sense-IT); and the last one included content from cognitive-behavioral therapy for crisis intervention (B.RIGHT). All of them obtained data supporting their acceptance and/or usability. In addition, two of them also proved their feasibility (DBT Coach and EMOTEO), and three also included a web server (overview screen) for therapists and/or patients (B.RIGHT, Sense-IT, mDiary app). (2) Wearable biosensor devices. Except for the study referred to above by Derks et al. [21], no software with clinical research was found.

Internet-Based Interventions. (1) Website-based interventions. There were two types of software (both beta versions) with some kind of clinical evidence (RDL: emerging). One of them, PRIOVI, was a guided self-management intervention based on schema therapy and to be used as an adjunctive intervention. PRIOVI reported data proving its feasibility and acceptance $[26,27]$. The other software tested the effectiveness of a DBT psychoeducation program as an unguided self-management intervention. A randomized controlled trial showed that the webbased intervention conferred additional and sustained improvements in BPD symptoms at 1-year followup when compared with a wait-list control group [28]. 
Table 2. Clinical research in technology-based psycho interventions for BPD

\begin{tabular}{|c|c|c|c|c|c|c|c|}
\hline $\begin{array}{l}\text { Type of } \\
\text { technology }\end{array}$ & $\begin{array}{l}\text { Project name and } \\
\text { reference }\end{array}$ & $\begin{array}{l}\text { Type of treatment and target } \\
\text { symptom }\end{array}$ & Distinctive features & $\begin{array}{l}\text { Feasibility, usability, and } \\
\text { acceptance testing }\end{array}$ & $\begin{array}{l}\text { Efficacy and effectiveness } \\
\text { testing }\end{array}$ & $\begin{array}{l}\text { Total } \\
\text { score }^{6}\end{array}$ & $\mathrm{RDL}^{6}$ \\
\hline \multirow[t]{5}{*}{ Mobile apps } & $\begin{array}{l}\text { B.RIGHT (Frías et al. } \\
{[20], 2020 \text {, Spain) }}\end{array}$ & $\begin{array}{l}\text { crisis intervention for emotional } \\
\text { crises }\end{array}$ & $\begin{array}{l}\text { beta version ( } 1 \text { point); cognitive- } \\
\text { behavioral-based intervention; includes a } \\
\text { chatbot in the mobile app and a web } \\
\text { server (overview screen) for therapists; } \\
\text { unguided self-management intervention }\end{array}$ & $\begin{array}{l}\text { pilot study with } 25 \text { patients } \\
\left.\text { (0.5 points); U: good }{ }^{1} \text { ( } 1 \text { point }\right)\end{array}$ & none & 2.5 & immature \\
\hline & $\begin{array}{l}\text { Sense-IT (Derks et al. } \\
{[21], 2019 \text {, The }} \\
\text { Netherlands) }\end{array}$ & $\begin{array}{l}\text { biofeedback (heart rate) for } \\
\text { emotional dysregulation }\end{array}$ & $\begin{array}{l}\text { beta version ( } 1 \text { point); biofeedback-based } \\
\text { intervention; includes wearable device } \\
\text { (smartwatch) and a web server (overview } \\
\text { screen) for patient/therapist; unguided } \\
\text { self-management intervention }\end{array}$ & $\begin{array}{l}\text { multiple user groups (patients, } \\
\text { therapists, expert users; } \\
1 \text { point); U: good for all types } \\
\text { of user groups } s^{1,2} \text { ( } 1 \text { point) }\end{array}$ & none & 3 & immature \\
\hline & $\begin{array}{l}\text { mDiary app (Helweg- } \\
\text { Joergensen et al. [25], } \\
\text { 2019, Denmark) }\end{array}$ & $\begin{array}{l}\text { adjunctive (self-monitoring and } \\
\text { psychoeducation) to usual DBT } \\
\text { for emotional dysregulation and } \\
\text { self-harm }\end{array}$ & $\begin{array}{l}\text { beta version (1 point); DBT-based } \\
\text { intervention; includes a web server } \\
\text { (overview screen) for patient/therapist and } \\
\text { GPS; unguided self-management } \\
\text { intervention }\end{array}$ & $\begin{array}{l}\text { multiple user groups (patients, } \\
\text { therapists) ( } 1 \text { point); U: } \\
\text { acceptable }^{1} \text { (higher scores by } \\
\text { patients than by therapists, } \\
\text { and GPS considered invasive } \\
\text { by patients) ( } 1 \text { point) }\end{array}$ & none & 3 & immature \\
\hline & $\begin{array}{l}\text { DBT Coach (Rizvi et al. } \\
\text { [23], 2011, United } \\
\text { States*; Rizvi et al. [24], } \\
\text { 2016, United States**) }\end{array}$ & $\begin{array}{l}\text { adjunctive to usual DBT for } \\
\text { improving mindfulness skills } \\
\text { training for comorbid SUD* } \\
\text { and NSSI** }\end{array}$ & $\begin{array}{l}\text { *prototype, }{ }^{* *} \text { full version (in stores) } \\
\text { ( } 1 \text { point); DBT-based intervention; } \\
\text { unguided self-management intervention }\end{array}$ & $\begin{array}{l}\text { *multiple user groups } \\
\text { (patients, therapists; } 1 \text { point); } \\
\text { ** pilot study with } 16 \text { patients } \\
\text { (0.5 points); F: }{ }^{*} \text { decreased } \\
\text { urge to use substances after } \\
10-15 \text { days ( } 1 \text { point); } \\
\text { ** decreased urge to self-harm } \\
\text { and NSSI after } 6 \text { months } \\
\text { (1 point); U/A: }{ }^{*} \text { usable and } \\
\text { helpful for patients and } \\
\text { therapists }{ }^{3}\left(1 \text { point); }{ }^{* *} \text { usable }\right. \\
\text { and helpful, but not enjoyable } \\
\text { (1 point) }\end{array}$ & none & $\begin{array}{l}* 3 \\
* * 3.5\end{array}$ & immature \\
\hline & $\begin{array}{l}\text { EMOTEO (Prada et al. } \\
\text { [22], 2017, Switzerland) }\end{array}$ & $\begin{array}{l}\text { adjunctive to usual DBT for } \\
\text { regulating aversive tension }\end{array}$ & $\begin{array}{l}\text { full version (in stores; } 1 \text { point); DBT-based } \\
\text { intervention; unguided self-management } \\
\text { intervention }\end{array}$ & $\begin{array}{l}\text { pilot study with } 16 \text { patients } \\
\text { (women) ( } 0.5 \text { points); F: } \\
\text { decreased aversion tension } \\
\text { after } 6 \text { months ( } 1 \text { point); U/A: } \\
\text { usable and helpful }{ }^{3}(1 \text { point })\end{array}$ & none & 3.5 & immature \\
\hline \multirow[t]{2}{*}{ Web-based } & $\begin{array}{l}\text { not reported (Zanarini } \\
\text { et al. [28], 2018, United } \\
\text { States) }\end{array}$ & $\begin{array}{l}\text { DBT-psychoeducation for BPD } \\
\text { symptoms }\end{array}$ & $\begin{array}{l}\text { beta version ( } 1 \text { point); DBT-based } \\
\text { intervention; program is laid out like a } \\
\text { book; unguided self-management } \\
\text { intervention }\end{array}$ & none & $\begin{array}{l}\text { RCT on effectiveness } \\
\text { ( } 4 \text { points); sample: } \\
\text { symptomatic volunteers } \\
\text { (women) recruited } \\
\text { through ads by one } \\
\text { research center; arms: } \\
\text { web-based }(n=40) \text { vs. } \\
\text { waitlist }(n=40) \text {; } \\
\text { procedure: } 3 \text {-month } \\
\text { treatment }+1 \text {-year } \\
\text { follow-up; outcome: } \\
\text { additional and } \\
\text { maintained decline in } \\
\text { overall BPD symptoms }{ }^{4} \text {; } \\
\text { ES: medium }(\mathrm{OR}=0.05) ; \\
\text { attrition: }<5 \%\end{array}$ & 5 & emerging \\
\hline & $\begin{array}{l}\text { PRIOVI (Fassbinder et } \\
\text { al. [26], 2015, Germany*; } \\
\text { Jacob et al. [27], 2018, } \\
\text { The Netherlands**) }\end{array}$ & $\begin{array}{l}\text { adjunctive to usual and } \\
\text { individual ST for BPD } \\
\text { symptoms }\end{array}$ & $\begin{array}{l}\text { beta version (1 point); ST-based } \\
\text { intervention; includes a clinician-facing } \\
\text { interface; guided self-management } \\
\text { intervention }\end{array}$ & $\begin{array}{l}\text { *single-case study; }{ }^{* *} \text { multiple } \\
\text { user groups (patients, } \\
\text { therapists) ( } 1 \text { point); F: } \\
\text { *improved BPD symptoms, } \\
\text { functioning, and schema } \\
\text { modes after } 6 \text { months } \\
\text { (1 point); }{ }^{* *} \text { improved BPD } \\
\text { symptoms after } 12 \text { months } \\
\text { (1 point); A: **helpful for all } \\
\text { types of user groups }{ }^{2}(1 \text { point) }\end{array}$ & none & $\begin{array}{l}* 2 \\
* * 4\end{array}$ & $\begin{array}{l}{ }^{*} \text { immature; } \\
{ }^{* *} \text { emerging }\end{array}$ \\
\hline \multirow[t]{2}{*}{$\begin{array}{l}\text { Virtual } \\
\text { reality }\end{array}$} & $\begin{array}{l}\text { not reported (Falconer } \\
\text { et al. [29], 2017, United } \\
\text { Kingdom) }\end{array}$ & $\begin{array}{l}\text { adjunctive to usual group MBT } \\
\text { for improving mentalization }\end{array}$ & $\begin{array}{l}\text { beta version ( } 1 \text { point); MBT-based } \\
\text { intervention; includes virtual desktop and } \\
\text { avatar software; delivered in a clinic and } \\
\text { guided by a therapist }\end{array}$ & $\begin{array}{l}\text { pilot study with } 15 \text { patients } \\
(0.5 \text { points); F: mentalization } \\
\text { measures remained } \\
\text { unchanged after } 4 \text { sessions } \\
\left.\text { (1 point); A: helpful }{ }^{2} \text { ( } 1 \text { point }\right)\end{array}$ & none & 3.5 & immature \\
\hline & $\begin{array}{l}\text { not reported (Nararro- } \\
\text { Haro et al. [30], 2016, } \\
\text { Spain) }\end{array}$ & $\begin{array}{l}\text { adjunctive to usual DBT for } \\
\text { improving mindfulness skills } \\
\text { training }\end{array}$ & $\begin{array}{l}\text { beta version ( } 1 \text { point); DBT-based } \\
\text { intervention; includes goggles and } \\
\text { headphones; delivered in a clinic and } \\
\text { guided by a therapist }\end{array}$ & $\begin{array}{l}\text { single-case study; F: higher } \\
\text { mindfulness scores after } \\
4 \text { sessions (1 point) }\end{array}$ & none & 2 & immature \\
\hline
\end{tabular}


Table 2 (continued)

\begin{tabular}{|c|c|c|c|c|c|c|c|}
\hline $\begin{array}{l}\text { Type of } \\
\text { technology }\end{array}$ & $\begin{array}{l}\text { Project name and } \\
\text { reference }\end{array}$ & $\begin{array}{l}\text { Type of treatment and target } \\
\text { symptom }\end{array}$ & Distinctive features & $\begin{array}{l}\text { Feasibility, usability, and } \\
\text { acceptance testing }\end{array}$ & $\begin{array}{l}\text { Efficacy and effectiveness } \\
\text { testing }\end{array}$ & $\begin{array}{l}\text { Total } \\
\text { score }^{6}\end{array}$ & $\mathrm{RDL}^{6}$ \\
\hline \multirow[t]{2}{*}{$\begin{array}{l}\text { Computer- } \\
\text { assisted }\end{array}$} & $\begin{array}{l}\text { CACR (Vita et al. [31], } \\
\text { 2018, Italy) }\end{array}$ & $\begin{array}{l}\text { computer-based cognitive } \\
\text { remediation therapy for } \\
\text { neurocognitive impairments } \\
\text { and psychosocial dysfunction }\end{array}$ & $\begin{array}{l}\text { beta version ( } 1 \text { point); cognitive } \\
\text { remediation-based intervention; delivered } \\
\text { in a clinic and guided by a therapist }\end{array}$ & $\begin{array}{l}\text { pilot study with } 15 \text { (TAU + } \\
\text { CACR) vs. } 15 \text { (TAU) patients } \\
\text { (0.5 points); F: CARC } \\
\text { improved working memory } \\
\text { and functioning, but not BPD } \\
\text { symptoms after } 4 \text { months } \\
\text { (1 point) }\end{array}$ & none & 2.5 & immature \\
\hline & $\begin{array}{l}\text { CBST (Wolf et al. [32], } \\
\text { 2011, Germany) }\end{array}$ & $\begin{array}{l}\text { computer-based therapy } \\
\text { adjunctive to conventional } \\
\text { group DBT for facilitating the } \\
\text { learning process in skills } \\
\text { training }\end{array}$ & $\begin{array}{l}\text { beta version ( } 1 \text { point); DBT-based } \\
\text { intervention; includes a CD-ROM; } \\
\text { self-help program }\end{array}$ & $\begin{array}{l}\text { pilot study with } 13 \text { (skills } \\
\text { training group }+ \text { CBST) vs. } 11 \\
\text { (skills training group) patients } \\
\text { (0.5 points); F: CBST } \\
\text { improved skills acquisition } \\
\text { after } 6 \text { months ( } 1 \text { point) }\end{array}$ & none & 2.5 & immature \\
\hline fMRI & $\begin{array}{l}\text { not reported (Paret et al. } \\
\text { [33], 2016, Germany*; } \\
\text { Zaehringer et al. [34], } \\
\text { 2019, Germany**) }\end{array}$ & $\begin{array}{l}\text { real-time fMRI neurofeedback } \\
\text { training (amygdala } \\
\text { hemodynamic activity) for } \\
\text { emotional dysregulation }\end{array}$ & $\begin{array}{l}\text { full version ( } 1 \text { point); biofeedback-based } \\
\text { intervention; includes thermometer bar } \\
\text { for amygdala activation; delivered in a } \\
\text { clinic and guided by a therapist }\end{array}$ & $\begin{array}{l}\text { *pilot study with } 8 \text { patients } \\
(0.5 \text { points); F: fMRI } \\
\text { neurofeedback training } \\
\text { improved dissociation and } \\
\text { lack of emotional awareness } \\
\text { after } 4 \text { sessions ( } 1 \text { point) }\end{array}$ & $\begin{array}{l}\text { *open trial on efficacy } \\
\text { ( } 3 \text { points); sample: } 26 \\
\text { patients (women) } \\
\text { recruited by one research } \\
\text { center; arm: fMRI } \\
\text { neurofeedback training; } \\
\text { procedure: } 4 \text {-session } \\
\text { treatment + } 1.5 \text {-month } \\
\text { follow-up; outcome: } \\
\text { maintained decline in } \\
\text { overall BPD symptoms } \\
\text { and emotional } \\
\text { dysregulation }{ }^{4,5} \text { ES: } \\
\text { medium (eta': } 0.14-0.18 \text { ); } \\
\text { attrition: } 2 \text { people }^{2}\end{array}$ & $\begin{array}{l}* 2.5 \\
{ }^{* *} 4\end{array}$ & $\begin{array}{l}{ }^{*} \text { immature; } \\
{ }^{* *} \text { emerging }\end{array}$ \\
\hline
\end{tabular}

A, acceptance; BPD, borderline personality disorder; CACR, computer-assisted cognitive remediation; CBST, computer-based skills training; CD-ROM, compact disc with read-only memory; DBT, dialectical behavior therapy; ES, effect size; F, feasibility; fMRI, functional magnetic resonance imaging; GPS, Global Positioning System; MBT, mentalization-based therapy; NSSI, nonsuicidal self-injury; OR, odds ratio; RCT, randomized controlled trial; RDL, research developmental level; ST, schema therapy; SUD, substance use disorder; TAU, treatment as usual; U, usability. ${ }^{1}$ Subjective Usability Scale. ${ }^{2}$ Qualitative interviews. ${ }^{3}$ Satisfaction and Usability Survey. ${ }^{4}$ Zanarini Rating Scale for Borderline Personality Disorder. ${ }^{5}$ Emotion Regulation Scale. ${ }^{6}$ RDL (scores): immature (0-3), emerging (4-8), promising (9-12), adequate (13-16), and validated (17-20).

(2) Virtual therapeutic communities. No software with clinical research was found.

Telehealth. (1) Videoconferencing. No software with clinical research was found. (2) Email messages. No software with clinical research was found.

\section{Psychosocial Interventions Based on Computing \\ Technology}

Virtual and Augmented Reality. There were two types of virtual reality software (beta version) with little clinical evidence (RDL: immature). One was an adjunctive mentalization-based therapy whose data proved its acceptance, but feasibility was not completely confirmed (outcome measures remained unchanged after the four sessions [29]); the other was an adjunctive DBT-based therapy that showed preliminary feasibility in a singlecase study [30].

Serious Games. No software with clinical research was found.

Eye-Tracking. No software with clinical research was found.

Computer-Based Interventions. There were two types of software (beta version) with little clinical evidence
(RDL: immature): cognitive-based cognitive remediation therapy proved its feasibility as an adjunctive therapy to treatment as usual (TAU) [31], and a DBT-based therapy showed its feasibility as an adjunctive intervention to conventional DBT-based treatment [32].

\section{Psychosocial Interventions Based on Computational} Technology

Artificial Intelligence Algorithms. No software with clinical research was found.

Machine Learning and Big Data. No software with clinical research was found.

Deep Learning and Neural Networks. No software with clinical research was found.

\section{Psychosocial Interventions Based on Medical \\ Technology}

Functional Magnetic Resonance Imaging. There was one type of software (full version) with some kind of clinical evidence (RDL: emerging). Initially, real-time fMRI neurofeedback training for amygdala hemodynamic activity was found to be feasible [33]. Then, the same research group performed an open trial showing sustained 
improvements in BPD symptoms at 1.5-month follow-up after using the same fMRI neurofeedback training [34].

Other Medical Technology. No other medical software with clinical research was found.

\section{Discussion}

\section{Summary of Evidence}

This scoping review aimed to delineate an up-to-date description of the ongoing technology-based psychosocial treatments for BPD with some kind of clinical research: (1) almost two-thirds of the studies (9/15) tested software explicitly conceived as adjunctive interventions to conventional therapy; (2) nearly half of the studies (7/15) were referred to as DBT-based software; (3) most studies (13/15) were focused on the initial stage of the clinical research cycle (feasibility/acceptance/usability testing), reporting good results at this point; (4) more than one-third of the studies (6/15) tested mobile apps; (5) clinical evidence was stronger for studies on webbased interventions than in studies on other technology; (6) there was no computational technology-based clinical research; and (7) there was no satisfaction/preference, security/safety, or efficiency testing for any software.

\section{Limitations}

Studies on feasibility, acceptance, and usability had several limitations. (1) Four studies only employed a user-centered approach instead of multiple user groups for usability and acceptance testing [20, 22, 24, 29]. Specifically, provider (therapist) opinions should be always taken into consideration because they are responsible of implementing the new technology in the healthcare setting [25]. (2) None of the studies assessed baseline cognitive intelligence as a potential moderator variable associated with the referred outcomes [35]. (3) Generalization of findings was limited because most samples predominantly consisted of women and two studies were based on single-case studies $[26,30]$.

\section{Recommendations for Future Research}

Future Research on Existing Technologies. Studies on effectiveness and efficacy are still pending in all software with prior data from feasibility, acceptance, and/or usability testing [36]. For those adjunctive interventions, these types of studies should include a combination therapy (TAU + technology-based treatment) and compare it with TAU alone. For those alternative interventions, studies should first compare the technology-based inter- vention with an inactive control group (waitlist). In addition, these studies on effectiveness and efficacy should include data from satisfaction/preference and safety/security testing. Particularly, mobile app-based psychosocial treatments for BPD need further empirical evidence beyond the current studies [37]. Studies on efficiency testing were obviously lacking and should be implemented because healthcare institutions with BPD patients as part of their clinical services may prioritize cost-effective software. This scoping review also supports the prioritization of Internet-based interventions, not only due to the current empirical evidence, but also because they are more scalable and useful than other promising technologies (e.g., fMRI feedback) from a public health perspective.

Proposed New Approaches to Technology-Based Psychosocial Treatments in BPD. Regardless of the findings of the current scoping review and considering prior research using technology for other mental health problems than BPD, several symptom-specific treatments for BPD are proposed. First, virtual reality may be used in order to induce positive emotions in BPD patients when depressed or when dealing with comorbid disorders such as posttraumatic stress disorder or agoraphobia [11]. Second, telehealth may be suitable for BPD patients who are living in remote places or during confinement [38]; virtual therapeutic communities may be pertinent for treatment-naïve patients or those socially isolated as a way to offer psychoeducation and emotional support, as long as these Internet forums are moderated by a trained clinician and participants have already been diagnosed with BPD rather than self-diagnosed [39]. To these ends, there is a need for integrative technology and scalability. For instance, some technology used for assessment may also be incorporated for intervention in BPD (e.g., eye-tracking, fMRI) $[40,41]$. Likewise, adaptations of proved technology in other mental disorders (e.g., mDiary for bipolar disorder) may be considered. Similarly, transdiagnostic software (e.g., for self-harm) may be valuable for treating patients with BPD [25, 42]. Ultimately, convergences between different but complementary technology should be prioritized (e.g., wearable devices plus mobile apps; virtual reality plus Internet-based) [21]. Specifically, the addition of serious games into mobile app-based and Internet-based interventions is warranted in order to promote real-world utilization (user engagement) of the former software, taking into account that these patients are prone to boredom susceptibility [13]. An alternative approach may be the gamification of this software by including gamified chatbots, which somehow partly replace therapeutic alliance 
as a core element for patients with special needs of interpersonal dependency [43].

Proposed Contraindications to Technology-Based Psychosocial Interventions in BPD. Due to the lack of safety and security testing, the following potential contraindications should be considered in people with BPD treated with technology-based psychosocial interventions. These contradictions do not emerge from the included studies, but are developed from other research on Internet interventions on other mental disorders. (1) Mobile apps and web-based treatments should be delivered cautiously with those at risk or already having comorbid behavioral addictions such as nomophobia, Internet addiction, and/ or gaming disorder [44-46]. (2) Virtual therapeutic communities should be avoided or monitored in those with higher baseline hostility levels, who in turn are at greater risk of online peer rejection and also cyberbullying [47, 48]. (3) Virtual reality should be implemented cautiously for those with higher baseline dissociation levels due to the immersion and absorption effects of this technology [49]. (4) Wearable biofeedback devices should be specially monitored in those with higher baseline neuroticism levels and trait anxiety because of the risk of paradoxical reactions (anxiety) and compulsive behaviors (reassurance). (5) The use of screen view monitors for therapists (connected to mHealth devices) should always be discussed with patients in general and those with greater paranoid ideation specifically, who may feel that such treatments are invasive. In particular, this subgroup has special "psychoeducation" needs regarding confidentiality and data protection/security [50]. In order to prevent these potential adverse effects, artificial intelligencebased assessment software may delineate high-risk subgroups on the basis of the aforementioned baseline clinical variables (behavioral addictions, dissociation, hostility, paranoid ideation, trait anxiety). Ultimately, this may lead to personalized technology-based psychosocial treatments in BPD [41].

\section{Conclusions}

This scoping review has shown that there are a growing and ongoing, but still incipient number of technology-based psychosocial interventions for BPD supported by some kind of clinical research and evidence. Particularly, there is emerging evidence for Internet-based interventions and real-time fMRI biofeedback, but only little evidence for mHealth interventions, virtual and augmented reality, and computer-based interventions. Ef- forts should be made to complete the clinical research cycle for this technology on the basis of both user (patient) and provider (therapist) recommendations. Ultimately, these types of interventions should be symptomspecific and solve real-world problems with an adequate and personalized risk-benefit balance.

\section{Conflict of Interest Statement}

Á. Frías received grants from the CaixaImpulse program (LCF/ TR/CI18/50030016), the Fundació Blanquerna-University of Ramon-Llull, and the Col-legi Oficial de Psicologia de Catalunya for the technical development of the mobile-app B-RIGHT. For the remaining authors non conflicts of interest are declared.

\section{Funding Sources}

Á. Frías received funds (SLT008/18/00175) for editing and proofreading the manuscript from the Pla Estratègic de Recerca i Innovació en Salut (PERIS 2019-2021) of the Department of Health, Generalitat de Catalunya. For the remaining authors no funding is declared.

\section{Author Contributions}

Á. Frías wrote the paper with L. Solves. S. Navarro, A. Riera, M. Hernández, and M. Antón drafted the manuscript. All authors contributed to the reviewing of the final version of the manuscript.

\section{References}

1 American Psychiatric Association. Diagnostic and Statistical Manual of Mental Disorders. 5th ed. Arlington (VA): American Psychiatric Association; 2013.

2 ten Have M, Verheul R, Kaasenbrood A, van Dorsselaer S, Tuithof M, Kleinjan M, et al Prevalence rates of borderline personality disorder symptoms: A study based on the Netherlands Mental Health Survey and Incidence Study-2. BMC Psychiatry. 2016;16(1):249.

3 Soloff $\mathrm{PH}$, Chiappetta L. Time, age, and predictors of psychosocial outcome in borderline personality disorder. J Pers Disord. 2020 Apr; 34(2):145-60.

4 Wagner T, Fydrich T, Stiglmayr C, Marschall P, Salize HJ, Renneberg B, et al. Societal costof-illness in patients with borderline personality disorder one year before, during and after dialectical behavior therapy in routine outpatient care. Behav Res Ther. 2014;61:1222.

5 Levy KN, McMain S, Bateman A, Clouthier T. Treatment of borderline personality disorder. Psychiatr Clin North Am. 2018;41(4):711-28. 
6 Stoffers JM, Völlm BA, Rücker G, Timmer A, Huband N, Lieb K. Psychological therapies for people with borderline personality disorder. Cochrane Database Syst Rev. 2012; 8:CD005652.

7 Arntz A, Stupar-Rutenfrans S, Bloo J, van Dyck R, Spinhoven P. Prediction of treatment discontinuation and recovery from borderline personality disorder: results from an RCT comparing schema therapy and transference focused psychotherapy. Behav Res Ther. 2015;74:60-71.

8 Temes CM, Zanarini MC. Recent developments in psychosocial interventions for borderline personality disorder. F1000Res. 2019 Apr;8:F1000 Faculty Rev-561.

9 Bakker D, Kazantzis N, Rickwood D, Rickard $\mathrm{N}$. Mental health smartphone apps: review and evidence-based recommendations for future developments. JMIR Ment Health. 2016; 3(1):e7.

10 Ben-Zeev D. Technology-based interventions for psychiatric illnesses: improving care, one patient at a time. Epidemiol Psychiatr Sci. 2014;23(4):317-21.

11 Freeman D, Reeve S, Robinson A, Ehlers A, Clark D, Spanlang B, et al. Virtual reality in the assessment, understanding, and treatment of mental health disorders. Psychol Med. 2017;47(14):2393-400.

12 Graham S, Depp C, Lee EE, Nebeker C, Tu X, $\mathrm{Kim} \mathrm{HC}$, et al. Artificial intelligence for mental health and mental illnesses: an overview. Curr Psychiatry Rep. 2019;21(11):116.

13 Cuijpers P, Noma N, Karyotaki E, Cipriani A, Furukawa TA. Effectiveness and acceptability of cognitive behavior therapy delivery formats in adults with depression: A network meta-analysis. JAMA Psychiatry. 2019;76(7): 700-7.

14 Lau HM, Smit JH, Fleming TM, Riper H. Serious games for mental health: are they accessible, feasible, and effective? A Systematic review and meta-analysis. Front Psychiatry. 2017;7:209.

15 Lawes-Wickwar S, McBain H, Mulligan K. Application and effectiveness of telehealth to support severe mental illness management: systematic review. JMIR Ment Health. 2018; 5(4):e62.

16 Mehrotra S, Tripathi R. Recent developments in the use of smartphone interventions for mental health. Curr Opin Psychiatry. 2018; 31(5):379-88.

17 Lezama-Nicolás R, Rodríguez-Salvador $\mathrm{M}$, Río-Belver R, Bildosola I. A bibliometric method for assessing technological maturity: the case of additive manufacturing. Scientometrics. 2018;117(3):1425-52.

18 Chauhan R, Singh I. Latest research and development on software testing techniques and tools. Int J Curr Eng Technol. 2014;4:236872.

19 Guyatt G, Cairns J, Churchill D, et al. Evidence-based medicine. A new approach to teaching the practice of medicine. JAMA. 1992;268(17):2420-5.
20 Frías Á, Palma C, Salvador A, Aluco E, Navarro $\mathrm{S}$, Farriols N, et al. B.RIGHT: usability and satisfaction with a mobile app for selfmanaging emotional crises in patients with borderline personality disorder. Australas Psychiatry. 2020 doi: 10.1177/ 1039856220924321 [Epub ahead of print]

21 Derks YP, Klaassen R, Westerhof GJ, Bohlmeijer ET, Noordzij L. Development of an ambulatory biofeedback app to enhance emotional awareness in patients with borderline personality disorder: Multicycle usability testing study. JMIR Mhealth Uhealth. 2019; 7:e13479.

22 Prada P, Zamberg I, Bouillault G, Jimenez N, Zimmermann J, Hasler R, et al. EMOTEO: A smartphone application for monitoring and reducing aversive tension in borderline personality disorder patients: A pilot study. Perspect Psychiatr Care. 2017;53(4):289-98.

23 Rizvi SL, Dimeff LA, Skutch J, Carroll D, Linehan MM. A pilot study of the DBT coach: an interactive mobile phone application for individuals with borderline personality disorder and substance use disorder. Behav Ther. 2011; 42(4):589-600.

24 Rizvi SL, Hughes CD, Thomas MC. The DBT Coach mobile application as an adjunct to treatment for suicidal and self-injuring individuals with borderline personality disorder: A preliminary evaluation and challenges to client utilization. Psychol Serv. 2016;13(4): 380-8.

25 Helweg-Joergensen S, Schmidt T, Lichtenstein MB, Pedersen SS. Using a mobile diary app in the treatment of borderline personality disorder: mixed methods feasibility study. JMIR Form Res. 2019;3(3):e12852.

26 Fassbinder E, Hauer A, Schaich A, Schweiger U, Jacob GA, Arntz A. Integration of e-health tools into face-to-face psychotherapy for borderline personality disorder: A chance to close the gap between demand and supply? J Clin Psychol. 2015;71(8):764-77.

27 Jacob GA, Hauer A, Köhne S, Assmann N, Schaich A, Schweiger U, et al. A schema therapy-based eHealth program for patients with borderline personality disorder (priovi): naturalistic single-arm observational study. JMIR Ment Health. 2018;5(4):e10983.

28 Zanarini MC, Conkey LC, Temes CM, Fitzmaurice GM. Randomized controlled trial of web-based psychoeducation for women with borderline personality disorder. J Clin Psychiatry. May-Jun 2018;79(3):16m11153.

29 Falconer CJ, Cutting P, Bethan Davies E, Hollis C, Stallard P, Moran P. Adjunctive avatar therapy for mentalization-based treatment of borderline personality disorder: A mixedmethods feasibility study. Evid Based Ment Health. 2017;20(4):123-7.

30 Nararro-Haro MV, Hoffman HG, Garcia-Palacios A, Sampaio M, Alhalabi W, Hall K, et al. The use of virtual reality to facilitate mindfulness skills training in dialectical behavioral therapy for borderline personality disorder: a case study. Front Psychol. 2016 Nov;7:1573.
31 Vita A, Deste G, Barlati S, Poli R, Cacciani P, De Peri L, et al. Feasibility and effectiveness of cognitive remediation in the treatment of borderline personality disorder. Neuropsychol Rehabil. 2018;28(3):416-28.

32 Wolf M, Ebner-Priemer U, Schramm E, Domsalla M, Hautzinger M, Bohus M. Maximizing skills acquisition in dialectical behavioral therapy with a CD-ROM-based self-help program: results from a pilot study. Psychopathology. 2011;44(2):133-5.

33 Paret C, Kluetsch R, Zaehringer J, Ruf M, Demirakca T, Bohus M, et al. Alterations of amygdala-prefrontal connectivity with realtime fMRI neurofeedback in BPD patients. Soc Cogn Affect Neurosci. 2016;11(6):95260.

34 Zaehringer J, Ende G, Santangelo P, Kleindienst N, Ruf M, Bertsch K, et al. Improved emotion regulation after neurofeedback: A single-arm trial in patients with borderline personality disorder. Neuroimage Clin. 2019; 24:102032.

35 Dubad M, Winsper C, Meyer C, Livanou M Marwaha S. Systematic review of the psychometric properties, usability and clinical impacts of mobile mood-monitoring applications in young people. Psychol Med. 2018; 48(2):208-28.

36 Klein JP, Hauer A, Berger T, Fassbinder E, Schweiger U, Jacob G. Protocol for the REVISIT-BPD Trial: A randomized controlled trial testing the effectiveness of an Internetbased self-management intervention in the treatment of borderline personality disorder (BPD). Front Psychiatry. 2018;9:439.

37 Ilagan GS, Iliakis EA, Wilks CR, Vahia IV, Choi-Kain LW. Smartphone applications targeting borderline personality disorder symptoms: a systematic review and meta-analysis. Borderline Personal Disord Emot Dysregul. 2020 Jun;7(1):12

38 Munz D, Jansen A, Böhmig C. Remote treatment from a psychotherapeutic point of view. Fortschr Neurol Psychiatr. 2019;87:165-71.

39 Habermeyer E, Habermeyer V, Jähn K, Domes G, Nagel E, Herpertz SC. An Internet based discussion board for persons with borderline personality disorders moderated health care professionals. Psychiatr Prax. 2009;36:23-9. German.

40 Bertsch K, Krauch M, Stopfer K, Haeussler K, Herpertz SC, Gamer M. Interpersonal threat sensitivity in borderline personality disorder: an eye-tracking study. J Pers Disord. 2017; 31(5):647-70.

41 Schmitgen MM, Niedtfeld I, Schmitt R, Mancke F, Winter D, Schmahl C, et al. Individualized treatment response prediction of dialectical behavior therapy for borderline personality disorder using multimodal magnetic resonance imaging. Brain Behav. 2019; 9(9): 01384. 
42 Wilks CR, Lungu A, Ang SY, Matsumiya B, Yin Q, Linehan MM. A randomized controlled trial of an Internet delivered dialectical behavior therapy skills training for suicidal and heavy episodic drinkers. J Affect Disord. 2018;232:219-28.

43 Bedics JD, Atkins DC, Harned MS, Linehan MM. The therapeutic alliance as a predictor of outcome in dialectical behavior therapy versus nonbehavioral psychotherapy by experts for borderline personality disorder. Psychotherapy (Chic). 2015;52(1):67-77.

44 Chen TH, Hsiao RC, Liu TL, Yen CF. Predicting effects of borderline personality symptoms and self-concept and identity disturbances on internet addiction, depression, and suicidality in college students: A prospective study. Kaohsiung J Med Sci. 2019;35(8):50814.
$45 \mathrm{Lu} \mathrm{WH}$, Lee KH, Ko CH, Hsiao RC, Hu HF, Yen CF. Relationship between borderline personality symptoms and Internet addiction: the mediating effects of mental health problems. J Behav Addict. 2017;6(3):434-41.

46 Marmet S, Studer J, Wicki M, Bertholet N, Khazaal Y, Gmel G. Unique versus shared associations between self-reported behavioral addictions and substance use disorders and mental health problems: A commonality analysis in a large sample of young Swiss men. J Behav Addict. 2019;8(4):664-77.

47 Lazarus SA, Cheavens JS. An examination of social network quality and composition in women with and without borderline personality disorder. Pers Disord. 2017;8(4):340-8.
48 Richmond JR, Edmonds KA, Rose JP, Gratz KL. Examining the impact of online rejection among emerging adults with borderline personality pathology: development of a novel online group chat social rejection paradigm. Pers Disord. 2020;11(5):301-11.

49 Aardema F, O’Connor K, Côté S, Taillon A. Virtual reality induces dissociation and lowers sense of presence in objective reality. Cyberpsychol Behav Soc Netw. 2010;13(4):42935.

50 Orme W, Bowersox L, Vanwoerden S, Fonagy $\mathrm{P}$, Sharp C. The relation between epistemic trust and borderline pathology in an adolescent inpatient sample. Borderline Personal Disord Emot Dysregul. 2019;6(1):13. 